\title{
Topological damping of Aharonov-Bohm effect: quantum graphs and vertex conditions
}

\author{
P. Kurasov and A. Serio \\ Department of Mathematics, Stockholm University, \\ 10691 Stockholm, Sweden \\ kurasov@math.su.se
}

\section{PACS 02.30.Tb, 03.65.Vf, 05.60.Gg DOI 10.17586/2220-8054-2015-6-3-309-319}

The magnetic Schrödinger operator was studied on a figure 8-shaped graph. It is shown that for specially chosen vertex conditions, the spectrum of the magnetic operator is independent of the flux through one of the loops, provided the flux through the other loop is zero. Topological reasons for this effect are explained.

Keywords: Quantum graphs, Magnetic field, Trace formula.

Received: 2 April 2015

\section{Introduction}

Transport of quantum particles in nanostructures can be described by Schrödinger equation on metric graphs, also known as quantum graphs. The spectral theory of quantum graphs is a hot topic in modern mathematical physics and many interesting properties have been observed and described, see for example recent monographs on the subject $[2,9,12]$. Propagation of particles in such structures is described by certain matching conditions at the vertices and by electric and magnetic potentials on the edges. The vertex conditions reflect the topology of the graph, connecting together limiting values of functions at one particular vertex. Transport along the edges is governed by the electric potential and is reminiscent of the one-dimensional Schrödinger equation. The magnetic potential plays a role only if the underlying graph contains cycles and the flux of the magnetic field through the cycles is not multiple of $2 \pi[4,8]$. Such dependence of the spectrum on the magnetic fluxes is usually understood as the Aharonov-Bohm effect, which goes back to [1]. The intuition behind Aharonov-Bohm effect is that a charged particle going around cycles 'feels' the magnetic field inside the cycles. If magnetic flux is a multiple of $2 \pi$, then the magnetic potential can be removed and therefore does not influence the spectrum.

In the current article, we consider a figure 8-shaped graph with magnetic Schrödinger operator. Our intuition, based on Aharonov-Bohm effect, tells us that the spectrum depends on the magnetic field fluxes through the two loops; explicit calculations support this prediction. On the other hand, it is discovered that if one of the fluxes is zero, then the spectrum might be independent of the flux through the other loop. This fact appears surprising, since no difference to the original Aharonov-Bohm setting can easily be seen. We use trace formula connecting the spectrum of the operator to the set of periodic orbits on the underlying metric graph. It appears that only the orbits going around the loops with nontrivial flux in opposite directions, and therefore not feeling the flux, contribute to the trace formula. We call this observation the topological damping of the Aharonov-Bohm effect and discuss how to obtain other graphs exhibiting the same property. 


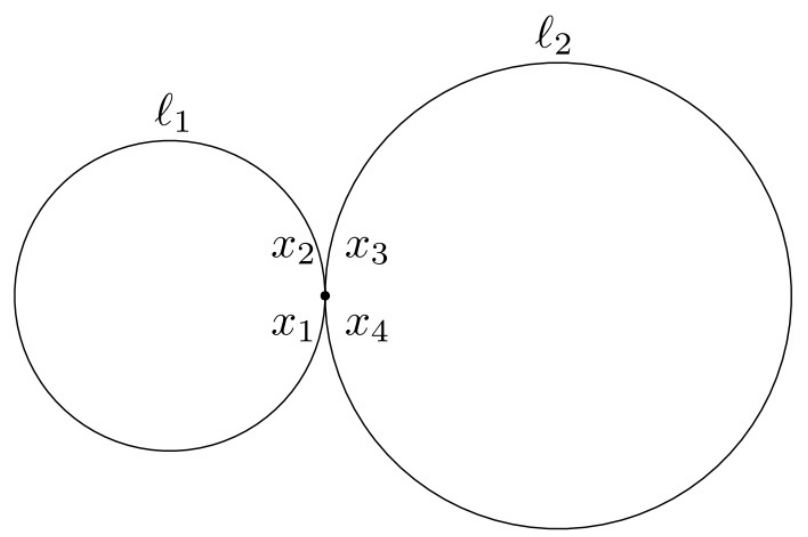

FIG. 1. The figure 8-shaped graph

The article is organized as follows: we first define the graph and the operator and calculate the spectrum explicitly. In the last section the trace formula is considered and the reason for the topological damping is explained. The role of vertex conditions is illuminated.

\section{Getting started}

Let us consider the metric figure 8 -shaped graph $\Gamma_{\infty}$ formed by two edges $E_{1}=$ $\left[x_{1}, x_{2}\right]$ and $E_{2}=\left[x_{3}, x_{4}\right]$, joined together at one vertex $V=\left\{x_{1}, x_{2}, x_{3}, x_{4}\right\}$.

We study the magnetic Schrödinger operator on $\Gamma_{\infty}$ :

$$
L=\left(i \frac{d}{d x}+a(x)\right)^{2},
$$

with zero electric potential and arbitrary integrable real magnetic potential $a$. To make the operator self-adjoint, we assume vertex matching conditions of the form:

$$
i(S-\mathbf{I}) \vec{u}=(S+\mathbf{I}) \partial \vec{u},
$$

where $\vec{u}$ and $\partial \vec{u}$ are the vectors of all limit values of the function and its normal extended derivatives at the vertex $V$ :

$$
\vec{u}=\left(\begin{array}{c}
u\left(x_{1}\right) \\
u\left(x_{2}\right) \\
u\left(x_{3}\right) \\
u\left(x_{4}\right)
\end{array}\right), \quad \partial \vec{u}=\left(\begin{array}{c}
u^{\prime}\left(x_{1}\right)-i a\left(x_{1}\right) u\left(x_{1}\right) \\
-\left(u^{\prime}\left(x_{2}\right)-i a\left(x_{2}\right) u\left(x_{2}\right)\right) \\
u^{\prime}\left(x_{3}\right)-i a\left(x_{3}\right) u\left(x_{3}\right) \\
-\left(u^{\prime}\left(x_{4}\right)-i a\left(x_{4}\right) u\left(x_{4}\right)\right)
\end{array}\right) .
$$

The matrix $S$ is unitary and is used to parametrize all possible matching conditions making the operator $L$ self-adjoint in $L_{2}\left(\Gamma_{\infty}\right)$ when defined on all functions from the Sobolev space $W_{2}^{2}(\Gamma \backslash V)$ satisfying $(2)$.

The spectrum of the operator $L$ is purely discrete and consists of eigenvalues accumulating towards $+\infty$. The eigenvalues can be calculated by solving the eigenvalue equation:

$$
\left(i \frac{d}{d x}+a(x)\right)^{2} \psi(x)=\lambda \psi(x)
$$

on each of the intervals and substituting the solution into the vertex conditions (2). Our main interest is the dependence of the spectrum upon the magnetic potential $a$. The first elementary observation is that magnetic potential can be eliminated on each of the edges, 
but not globally, leading to new vertex conditions that depend on the integrals of the magnetic potential along the two loops:

$$
\phi_{j}=\int_{x_{2 j-1}}^{x_{2 j}} a(x) d x, j=1,2 .
$$

It appears that that particular form of the magnetic potential does not play any role. The following transformation:

$$
U_{a}: u(x) \mapsto \exp \left(-i \int_{x_{2 j-1}}^{x} a(y) d y\right) u(x),
$$

maps the operator $L$ into the operator $\mathbf{L}=U_{a} L U_{a}^{-1}$ given by the same differential expression but with zero magnetic potential:

$$
\mathbf{L}=-\frac{d^{2}}{d x^{2}}
$$

on the set of functions from $W_{2}^{2}(\Gamma \backslash V)$ satisfying vertex conditions:

$$
i\left(S^{\phi_{1}, \phi_{2}}-\mathbf{I}\right) \vec{u}=\left(S^{\phi_{1}, \phi_{2}}+\mathbf{I}\right) \partial_{n} \vec{u},
$$

which are obtained from (2) by substituting the matrix $S$ with:

$$
S^{\phi_{1}, \phi_{2}}=D S D^{-1}, \quad D=\left(\begin{array}{cccc}
1 & 0 & 0 & 0 \\
0 & e^{-i \phi_{1}} & 0 & 0 \\
0 & 0 & 1 & 0 \\
0 & 0 & 0 & e^{-i \phi_{2}}
\end{array}\right)
$$

and the vector of extended derivatives $\partial \vec{u}$ - with the vector of normal derivatives

$$
\partial_{n} \vec{u}=\left(\begin{array}{c}
u^{\prime}\left(x_{1}\right) \\
-u^{\prime}\left(x_{2}\right) \\
u^{\prime}\left(x_{3}\right) \\
-u^{\prime}\left(x_{4}\right)
\end{array}\right)
$$

The integrals $\phi_{j}$ can be interpreted as fluxes of the magnetic field through the corresponding loops.

In general, the spectrum of the operator $\mathbf{L}$ depends on the fluxes, but it might happen that only the sum of the fluxes counts. For example, if $S=\left(\begin{array}{llll}0 & 0 & 0 & 1 \\ 0 & 0 & 1 & 0 \\ 0 & 1 & 0 & 0 \\ 1 & 0 & 0 & 0\end{array}\right)$, then the graph $\Gamma_{\infty}$ is equivalent to the loop of length $\mathcal{L}=x_{2}-x_{1}+x_{4}-x_{3}$ and the spectrum obviously depends on the sum of the of the fluxes $\phi_{1}+\phi_{2}$. This case is not interesting, since the anomalous behavior of the spectrum is due to the choice of the vertex conditions that do not respect the geometry of the graph; the vertex $V$ can be divided into two vertices, $V_{1}=\left\{x_{1}, x_{4}\right\}$ and $V_{2}=\left\{x_{2}, x_{3}\right\}$ and the vertex conditions separately connect the boundary values corresponding to the two new vertices. Such boundary conditions do not correspond to the figure 8-shaped graph, but rather to the loop graph. 
Another degenerate example is when $S=\left(\begin{array}{llll}0 & 1 & 0 & 0 \\ 1 & 0 & 0 & 0 \\ 0 & 0 & 0 & 1 \\ 0 & 0 & 1 & 0\end{array}\right)$. In this case, every second eigenvalue depends only on $\phi_{1}$ and all other - only on $\phi_{2}$. The vertex conditions connect together the pairs of end points $\left(x_{1}, x_{2}\right)$ and $\left(x_{3}, x_{4}\right)$ separately. The corresponding metric graph is not $\Gamma_{\infty}$, but rather two separate loops formed by the two edges. This case is not interesting for us either.

In what follows, we study the magnetic Schrödinger operator corresponding to the vertex conditions given by the following vertex scattering matrix:

$$
S=\left(\begin{array}{cccc}
0 & 0 & \alpha & \beta \\
0 & 0 & -\beta & \alpha \\
\alpha & -\beta & 0 & 0 \\
\beta & \alpha & 0 & 0
\end{array}\right), \quad \alpha, \beta \in \mathbb{R}, \alpha^{2}+\beta^{2}=1
$$

This unitary matrix connects together boundary values at all four end points and therefore is properly connecting. One may visualize this by the following picture, where all possible scattering processes are indicated by curves. It will be shown that interesting effects can

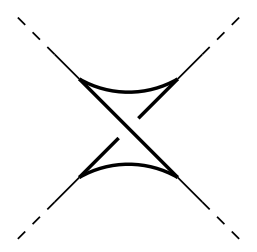

FIG. 2. Visual representation of the connections given by the vertex conditions with $S$ given by (9): the curves indicate possible passages.

be observed if the probabilities of these passages are equal, which corresponds to the choice $\alpha=\beta=1 / \sqrt{2}$ :

$$
S=\left(\begin{array}{cccc}
0 & 0 & \frac{1}{\sqrt{2}} & \frac{1}{\sqrt{2}} \\
0 & 0 & -\frac{1}{\sqrt{2}} & \frac{1}{\sqrt{2}} \\
\frac{1}{\sqrt{2}} & -\frac{1}{\sqrt{2}} & 0 & 0 \\
\frac{1}{\sqrt{2}} & \frac{1}{\sqrt{2}} & 0 & 0
\end{array}\right)
$$

\section{Explicit calculation of the spectrum.}

Our immediate goal is to derive the equation describing the spectrum of the operator $\mathbf{L}$, depending on the fluxes $\phi_{1}$ and $\phi_{2}$ and parameters $\alpha$ and $\beta$. All nonzero eigenvalues of $\mathbf{L}$ can be calculated using the vertex and edge scattering matrices $[3,5,6,7,10]$. The matrix $S$, and therefore the matrix $S^{\phi_{1}, \phi_{2}}=D S D^{-1}$, appearing in the vertex conditions is not only unitary, but also Hermitian. It follows that the corresponding vertex scattering 
matrix $S_{v}$ does not depend on the energy and coincides with $S^{\phi_{1}, \phi_{2}}$ :

$$
S_{v} \equiv S^{\phi_{1}, \phi_{2}}=\left(\begin{array}{cccc}
0 & 0 & \alpha & e^{i \phi_{2}} \beta \\
0 & 0 & -e^{-i \phi_{1}} \beta & e^{-i\left(\phi_{1}-\phi_{2}\right)} \alpha \\
\alpha & -e^{i \phi_{1}} \beta & 0 & 0 \\
e^{-i \phi_{2}} \beta & e^{i\left(\phi_{1}-\phi_{2}\right)} \alpha & 0 & 0
\end{array}\right) .
$$

The differential operator on the edges does not contain any electric or magnetic potential, hence the plane waves penetrate the edges without any reflection but with the addition of extra phases. The corresponding edge scattering matrix is

$$
S_{e}=\left(\begin{array}{cccc}
0 & e^{i k \ell_{1}} & 0 & 0 \\
e^{i k \ell_{1}} & 0 & 0 & 0 \\
0 & 0 & 0 & e^{i k \ell_{2}} \\
0 & 0 & e^{i k \ell_{2}} & 0
\end{array}\right)
$$

where $\ell_{j}=x_{2 j}-x_{2 j-1}, j=1,2$ are the lengths of the edges. Then, all nonzero eigenvalues are given by the solutions of the equation:

$$
\operatorname{det}\left(S_{e}(k) S_{v}-\mathbb{I}\right)=0
$$

which is equivalent to:

$$
\begin{array}{r}
\operatorname{det}\left(\begin{array}{cccc}
-1 & 0 & -e^{i k \ell_{1}} e^{-i \phi_{1}} \beta & e^{i k \ell_{1}} e^{-i\left(\phi_{1}-\phi_{2}\right)} \alpha \\
0 & -1 & e^{i k \ell_{1}} \alpha & e^{i k \ell_{1}} e^{i \phi_{2}} \beta \\
e^{i k \ell_{2}} e^{-i \phi_{2}} \beta & e^{i k \ell_{2}} e^{i\left(\phi_{1}-\phi_{2}\right)} \alpha & -1 & 0 \\
e^{i k \ell_{2}} \alpha & -e^{i k \ell_{2}} e^{i \phi_{1}} \beta & 0 & -1
\end{array}\right)=0, \\
\Leftrightarrow 1+\left(\alpha^{2}+\beta^{2}\right)^{2} e^{2 i k\left(\ell_{1}+\ell_{2}\right)}+2\left(\cos \left(\phi_{1}+\phi_{2}\right) \beta^{2}-\cos \left(\phi_{1}-\phi_{2}\right) \alpha^{2}\right) e^{i k\left(\ell_{1}+\ell_{2}\right)}=0 .
\end{array}
$$

Taking into account that $\alpha^{2}+\beta^{2}=1$ and $e^{i k\left(\ell_{1}+\ell_{2}\right)} \neq 0$, we arrive at the following secular equation:

$$
\cos k\left(\ell_{1}+\ell_{2}\right)=\alpha^{2} \cos \left(\phi_{1}-\phi_{2}\right)-\beta^{2} \cos \left(\phi_{1}+\phi_{2}\right) \text {. }
$$

The right hand side of this equation is a constant between -1 and 1 and hence, solutions to the equation form two periodic in $k$ sequences. The corresponding eigenvalues, $\lambda=k^{2}$, in general, depend on both magnetic fluxes $\phi_{1}$ and $\phi_{2}$.

An interesting phenomenon occurs if one choses $\alpha=\beta=1 / \sqrt{2}$, i.e. the matrix $\mathbf{S}$ given by (10). The secular equation (16) takes the form:

$$
\begin{aligned}
\cos k\left(\ell_{1}+\ell_{2}\right) & =\frac{\cos \left(\phi_{1}-\phi_{2}\right)-\cos \left(\phi_{1}+\phi_{2}\right)}{2} \\
& =\sin \phi_{1} \sin \phi_{2} .
\end{aligned}
$$

It follows, that if one of the magnetic fluxes is an integer multiple of $\pi$, then the spectrum is independent of the other flux. This is a trivial consequence of the secular equation (17), but we are interested in having an intuitive explanation of this phenomena. The AharonovBohm effect tells us that the spectrum of a system, like the magnetic Schrödinger operator on $\Gamma_{\infty}$ should depend on the magnetic fluxes. This dependence is damped only in very special cases. Why is it so special when one of the fluxes is zero? An explicit answer to this question is given in the following section. We use the trace formula connecting the spectrum of a quantum graph to the set of periodic orbits on the underlying metric graph.

In what follows, we are interested in this special case, when one of the fluxes is zero. Without loss of generality, we may assume that $\phi_{1}=0$. 
First, let us determine whether $\lambda=0$ is an eigenvalue of the operator $\mathbf{L}$ or not. The value of $k=0$ is a solution to the secular equation only if $\sin \phi_{1} \sin \phi_{2}=1$. If one of the fluxes is zero, then $k=0$ is not a solution to the secular equation. It follows that the so-called algebraic multiplicity ${ }^{1} m_{a}(0)[10,6]$ of the zero eigenvalue is zero.

Let us turn to calculation of the spectral multiplicity $m_{s}(0)$ - the number of linearly independent solutions to the equation $\mathbf{L} \psi=0$. In order to emphasize that only the lengths of the edges are important, we parameterize the edges as follows:

$$
\left[x_{1}, x_{2}\right]=\left[0, \ell_{1}\right], \quad\left[x_{3}, x_{4}\right]=\left[0, \ell_{2}\right] .
$$

All solutions to the differential equation are then given by:

$$
\psi(x)= \begin{cases}a_{1} x+b_{1} & \text { if } x \in\left[x_{1}, x_{2}\right], \\ a_{2} x+b_{2} & \text { if } x \in\left[x_{3}, x_{4}\right] .\end{cases}
$$

Then, at the endpoints, we have:

$$
\vec{\psi}=\left(\begin{array}{c}
b_{1} \\
a_{1} l_{1}+b_{1} \\
b_{2} \\
a_{2} l_{2}+b_{2}
\end{array}\right), \quad \partial_{n} \vec{\psi}=\left(\begin{array}{c}
a_{1} \\
-a_{1} \\
a_{2} \\
-a_{2}
\end{array}\right) .
$$

The matrix $\mathbf{S}^{\phi_{1}, \phi_{2}}$ is unitary and Hermitian, hence its eigenvalues are just \pm 1 . Therefore, the vertex conditions (7) are satisfied if and only if both the left and right hand sides are equal to zero:

$$
\begin{aligned}
& \left(\begin{array}{cccc}
-\frac{1}{2} & 0 & \frac{1}{2 \sqrt{2}} & \frac{e^{-i \phi_{2}}}{2 \sqrt{2}} \\
0 & -\frac{1}{2} & -\frac{e^{i \phi_{1}}}{2 \sqrt{2}} & \frac{e^{i \phi_{1}-i \phi_{2}}}{2 \sqrt{2}} \\
\frac{1}{2 \sqrt{2}} & -\frac{e^{-i \phi_{1}}}{2 \sqrt{2}} & -\frac{1}{2} & 0 \\
\frac{e^{i \phi_{2}}}{2 \sqrt{2}} & \frac{e^{-i \phi_{1}+i \phi_{2}}}{2 \sqrt{2}} & 0 & -\frac{1}{2}
\end{array}\right) \quad\left(\begin{array}{c}
a_{1} \\
-a_{1} \\
a_{2} \\
-a_{2}
\end{array}\right)=0 \\
& \left(\begin{array}{cccc}
\frac{1}{2} & 0 & \frac{1}{2 \sqrt{2}} & \frac{e^{-i \phi_{2}}}{2 \sqrt{2}} \\
0 & \frac{1}{2} & -\frac{e^{i \phi_{1}}}{2 \sqrt{2}} & \frac{e^{i \phi_{1}-i \phi_{2}}}{2 \sqrt{2}} \\
\frac{1}{2 \sqrt{2}} & -\frac{e^{-i \phi_{1}}}{2 \sqrt{2}} & \frac{1}{2} & 0 \\
\frac{e^{i \phi_{2}}}{2 \sqrt{2}} & \frac{e^{-i \phi_{1}+i \phi_{2}}}{2 \sqrt{2}} & 0 & \frac{1}{2}
\end{array}\right) \quad\left(\begin{array}{c}
b_{1} \\
a_{1} l_{1}+b_{1} \\
b_{2} \\
a_{2} l_{2}+b_{2}
\end{array}\right)=0 .
\end{aligned}
$$

We omit tedious computations and give just a sketch. From the first equation, we obtain $a_{1}=a_{2}=0$, then plugging this result into the second equation we obtain $b_{1}=b_{2}=0$ for any values of $\phi_{2}$. This proves that $\lambda=0$ is not an eigenvalue and hence the spectral multiplicity (as well as the algebraic multiplicity) is zero in this case.

\footnotetext{
${ }^{1}$ The algebraic multiplicity is the order of zero in the secular equation.
} 
In summary, the spectrum of the magnetic Schrödinger operator on $\Gamma_{\infty}$ is given by the solutions of the secular equation:

$$
\cos k \mathcal{L}=0, \quad \mathcal{L}=\ell_{1}+\ell_{2},
$$

provided one of the magnetic fluxes is zero.

\section{Topological reasons for damping}

We have seen that in the case $\phi_{1}=0$ the spectrum does not depend on the flux $\phi_{2}$ - the Aharonov-Bohm effect is damped which contradicts our intuition. The main goal of this section is to explain that this effect has a topological explanation. We are going to use trace formula (see $[3,5,10,6,13]$ ) connecting the spectrum of a quantum graph to the set of periodic orbits on the metric graph. It will be shown that orbits that "feel" the magnetic flux $\phi_{2}$ give zero total contribution into the trace formula.

Under a periodic orbit, we understand any oriented closed path on the graph $\Gamma_{\infty}$, which is allowed to turn back at vertices only. Paths having opposite directions are considered to be different. The trace formula for the Laplace operator on a metric graph can be written as $[3,5,10,6,13]$ :

$$
\begin{gathered}
u(k):=2 m_{s}(0) \delta(k)+\sum_{k_{n} \neq 0}\left(\delta\left(k-k_{n}\right)+\delta\left(k+k_{n}\right)\right) \\
=\left(2 m_{s}(0)-m_{a}(0)\right) \delta(k)+\frac{\mathcal{L}}{\pi}+\frac{1}{\pi} \sum_{p \in \mathcal{P}} l(\operatorname{prim}(p)) S_{v}(p) \cos k l(p),
\end{gathered}
$$

where

- $\mathcal{L}=\ell_{1}+\ell_{2}$ is the total length of the graph,

- $m_{s}(0)$ is the (spectral) multiplicity of the eigenvalue zero;

- $m_{a}(0)$ is the algebraic multiplicity of the eigenvalue zero - the order of zero given by the secular equation (16);

- $p$ is a closed path on $\Gamma$;

- $\mathcal{P}$ is the set of closed paths;

- $l(p)$ is the length of the closed path $p$;

- $\operatorname{prim}(p)$ is one of the primitive paths for $p$;

- $S_{v}(p)$ is the product of all vertex scattering coefficients along the path $p$.

This formula was proven for the Laplace operator with standard vertex conditions (assuming that the functions are continuous and the sum of normal derivatives is zero), but just the same proof holds in the case where the vertex conditions are described by vertex scattering matrices which are independent of the energy [11]. In the considered case, the vertex scattering matrices $S_{v}$ are Hermitian, and therefore, independent of the energy.

The fluxes $\phi_{1}$ and $\phi_{2}$ are contained in the products $S_{v}(p)$, since the entries of $S_{v} \equiv S^{\phi_{1}, \phi_{2}}$ depend on the fluxes (see formula (11)). Therefore, it is natural to expect that the left side of the equation also depends on the fluxes. Conversely, the left side in (23) is determined by the spectrum of $\mathbf{L}$, which in the case $\phi_{1}=0$, is independent of $\phi_{2}$. More precisely, the spectrum is determined by $\cos k \mathcal{L}=0$ (we have already shown that $\lambda=0$ is not an eigenvalue in this case, $\left.m_{s}(0)=0\right)$ :

$$
k_{n}=\frac{\pi}{2 \mathcal{L}}+\frac{\pi}{\mathcal{L}} n, n=0,1,2,3, \ldots
$$


Then, the left side of the trace formula can be written as:

$$
\begin{aligned}
u(k) & =\sum_{k_{n} \neq 0}\left(\delta\left(k-k_{n}\right)+\delta\left(k+k_{n}\right)\right) \\
& =\sum_{n \in \mathbb{Z}} \delta\left(k-\left(\frac{\pi}{2 \mathcal{L}}+\frac{\pi}{\mathcal{L}} n\right)\right) \\
& =\sum_{m \in \mathbb{Z}} \delta\left(k-\frac{\pi}{2 \mathcal{L}} m\right)-\sum_{m \in \mathbb{Z}} \delta\left(k-\frac{\pi}{\mathcal{L}} m\right) .
\end{aligned}
$$

We use now the Poisson summation formula:

$$
\sum_{n \in \mathbb{Z}} \delta(x-T n)=\frac{1}{T} \sum_{m \in \mathbb{Z}} e^{-i 2 \pi \frac{m}{T} x}
$$

and rewrite the last expression as follows:

$$
u(k)=\sum_{n \in \mathbb{Z}} \delta\left(k-\left(\frac{\pi}{2 \mathcal{L}}+\frac{\pi}{\mathcal{L}} n\right)\right)=\frac{2 \mathcal{L}}{\pi} \sum_{m \in \mathbb{Z}} e^{-i 4 \mathcal{L} m k}-\frac{\mathcal{L}}{\pi} \sum_{m \in \mathbb{Z}} e^{-i 2 \mathcal{L} m k} .
$$

This formula represents the distribution $u(k)$ as a formal exponential series. This series is independent of $\phi_{2}$, while the series on the right side of (23) formally contains $\phi_{2}$, since $S_{v}(p)$ depends on the second flux. Let us examine the series over all periodic orbits in more detail in order to understand the reason why all terms containing $\phi_{2}$ cancel. It appears that the cause is mostly topological.

The algebraic multiplicity of the eigenvalue zero is zero $m_{a}=0(k=0$ is not a solution to $\cos k \mathcal{L}=0$ ) and the right hand side of trace formula can be written as:

$$
u(k)=\frac{\mathcal{L}}{\pi}+\frac{1}{\pi} \sum_{p \in \mathcal{P}} l(\operatorname{prim}(p)) S_{v}(p) \cos k l(p) .
$$

Let us first note that the sum in the trace formula contains contributions from the paths that go around the left and right loops an equal number of times. This is due to the fact that the coefficients $12,21,34$ and 43 in the vertex scattering matrix are zero:

$$
\left(S_{v}\right)_{12}=\left(S_{v}\right)_{21}=\left(S_{v}\right)_{34}=\left(S_{v}\right)_{43}=0 .
$$

Therefore, the length of each path with nontrivial $S_{v}(p)$ is an integer multiple of the total length $\mathcal{L}:=\ell_{1}+\ell_{2}$.

The sum over all paths is taken over all closed paths and $l(\operatorname{prim} p)$ is the length of the corresponding primitive path. It will be convenient for us to distinguish paths with different starting edges - the first edges the path comes across. Then, the sum $\sum_{p \in \mathcal{P}} l(\operatorname{prim}(p)) S_{v}(p) \cos k l(p)$ can be written as two sums - over the paths that go around the left loop first and over the paths that go around the right loop first:

$$
\begin{aligned}
u(k) & =\frac{\mathcal{L}}{\pi}+\frac{1}{\pi} \sum_{p \in \mathcal{P}} l(\operatorname{prim}(p)) S_{v}(p) \cos k l(p) \\
& =\frac{\mathcal{L}}{\pi}+\frac{\ell_{1}}{\pi} \sum_{p \in \mathbb{P}_{l}} S_{v}(p) \cos k l(p)+\frac{\ell_{2}}{\pi} \sum_{p \in \mathbb{P}_{r}} S_{v}(p) \cos k l(p),
\end{aligned}
$$

where $\mathbb{P}_{l, r}$ denote the sets of paths where paths with different starting edges are considered different. The lower indices $l$ and $r$ indicate whether the path goes around the left or the right loop first. Each of the two sums can be treated in a similar fashion.

Let us first consider the series $\sum_{p \in \mathbb{P}_{l}} S_{v}(p) \cos k l(p)$ over all paths starting by going into the left edge. After going around the left loop, the path should go around the right 
loop and then again around the left one: the left and right loops appear one after another. Every such path can be uniquely parameterized by a series of indices $\nu_{j}= \pm$, indicating whether the path goes around the left or right path in the positive $(+)$ (clockwise following the orientation of the edges) or negative $(-)$ (counterclockwise) direction. All odd indices correspond to the left loop, all even - to the right loop. The number of signs is even, which reflects the fact that every such path goes around the left and right loops equal number of times. For example the path indicted on Fig. 3 is parameterized as $(+,-,+,+)$.

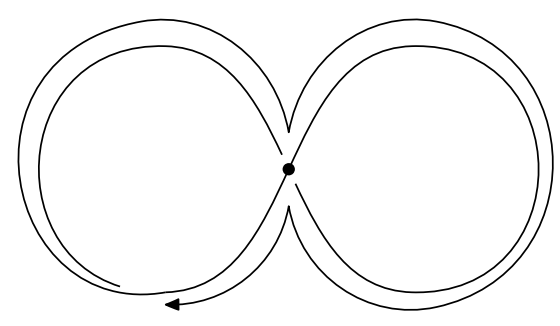

FIG. 3. A path of length $2\left(l_{1}+l_{2}\right)$.

Let us turn to the calculation of the coefficients $S_{v}(p)$. The path indicated on Fig. 3 has coefficient $S_{v}(p)$ equal to:

$$
\begin{aligned}
S_{v}(p) & =\left(S_{v}\right)_{14}\left(S_{v}\right)_{32}\left(S_{v}\right)_{13}\left(S_{v}\right)_{42} \\
& =e^{i \phi_{2}} \beta \cdot\left(-e^{-i \phi_{1}} \beta\right) \cdot \alpha \cdot e^{i\left(\phi_{1}-\phi_{2}\right)} \alpha \\
& =-e^{2 i \phi_{1}} \alpha^{2} \beta^{2}=\frac{-1}{4} e^{i 2 \phi_{1}} .
\end{aligned}
$$

One may calculate the same product using the original vertex scattering matrix (10), but taking into account that each time when the path goes along the left or right loop, the product gains the phase coefficient $e^{ \pm i \phi_{1}}$ or $e^{ \pm i \phi_{2}}$, respectively. The sign corresponds to positive or negative direction. Each time when the path crosses the middle vertex, $S_{v}(p)$ gets an extra term $\pm \frac{1}{\sqrt{2}}$. Note that only coefficients corresponding to the transitions $2 \rightarrow 3$ and $3 \rightarrow 2$ have a minus sign; all other coefficients are positive:

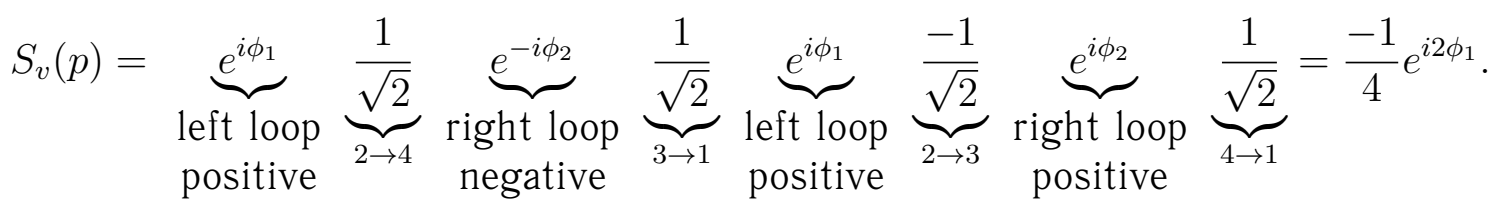

It will be convenient to see the product $S_{v}(p)$ corresponding to the path $\left(\nu_{1}, \nu_{2}, \ldots, \nu_{2 n}\right)$ divided into three factors

- the product of all phase factors

$$
e^{i \sum_{j=1}^{n} \nu_{2 j-1} \phi_{1}} \cdot e^{i \sum_{j=1}^{n} \nu_{2 j} \phi_{2}}
$$

- the product of absolute values of scattering coefficients

$$
\left(\frac{1}{\sqrt{2}}\right)^{2 n}=\frac{1}{2^{n}}
$$

- the product of sign factors \pm 1 . 
Our next claim is that only paths that every second time go around the right loop in a different direction give a contribution into the trace formula. Consider the path $p^{\prime}$ that contains the sequence $(\ldots, \underbrace{+}_{2 m}, \underbrace{+}_{2 m+1}, \underbrace{+}_{2 m+2} \ldots)$. Then, the contribution from the path $p^{\prime \prime}$ obtained from $p^{\prime}$ by reversing the edge with the number $2 m+1$, i.e. given by $(\ldots, \underbrace{+}_{2 m}, \underbrace{-}_{2 m+1}, \underbrace{+}_{2 m+2} \ldots)$, cancels the contribution from $p^{\prime}$. Really, the phase contributions from $p^{\prime}$ and $p^{\prime \prime}$ are the same, the absolute values are also the same, while the product of signs for $p^{\prime}$ contains $(-1) \times 1$ corresponding to transitions $3 \rightarrow 2$ and $1 \rightarrow 4$, in contrast to the product $1 \times 1$ appearing in the product for $p^{\prime \prime}$ (corresponds to the transitions $3 \rightarrow 1$ and $2 \rightarrow 4$, all other coefficients are the same). Similarly contributions from the paths given by $(\ldots, \underbrace{-}_{2 m}, \underbrace{-}_{2 m+1}, \underbrace{-}_{2 m+2} \ldots)$ and $(\ldots, \underbrace{-}_{2 m}, \underbrace{+}_{2 m+1}, \underbrace{-}_{2 m+2} \ldots)$ cancel each other.

We now assume that the path $p^{\prime}$ contains the sequence $(\ldots, \underbrace{+}_{2 m}, \underbrace{+}_{2 m+1}, \underbrace{-}_{2 m+2} \ldots)$, then the contribution from $p^{\prime \prime}$ corresponding to $(\ldots, \underbrace{+}_{2 m}, \underbrace{-}_{2 m+1}, \underbrace{-}_{2 m+2} \ldots)$ is just the same. It follows that only the paths of the form $\left(\nu_{1},+, \nu_{3},-, \nu_{5},+, \nu_{7},-, \ldots\right)$ and $\left(\nu_{1},-, \nu_{3},+, \nu_{5},-, \nu_{7},+, \ldots\right)$ survive in the series. Every such path has a discrete length (the number of edges it comes across) being multiple of 4 . The phase contribution from such paths is zero, since we assumed $\phi_{1}=0$. It follows that the sum over the periodic paths starting with the left loop does not depend on $\phi_{2}$. A similar result holds for the other sum, explaining the reason why the spectrum of the magnetic Schrödinger operator on $\Gamma_{\infty}$ does not depend on $\phi_{2}$, provided $\phi_{1}=0$.

Let us continue to calculate the sum over the periodic orbits. We have seen that only orbits of lengths $2 n \mathcal{L}$ (discrete length $4 n$ ) make a contribution to the series. Consider, for example, the orbits of length $2 \mathcal{L}$. Only the orbits of the form $\left(\nu_{1},+, \nu_{3},-\right)$ and $\left(\nu_{1},-, \nu_{3},+\right)$ give nonzero contributions. The phase contribution is $e^{i 0}=1$. The absolute value contribution is $\left(\frac{1}{\sqrt{2}}\right)^{4}=\frac{1}{4}$. The sign contribution is -1 . Together, there are $4 \times 2$ such orbits, since the signs $\nu_{1}, \nu_{3}$ can be chosen freely. So, the total contribution to the series is: $-\ell_{1} 2 \cos k 2 \mathcal{L}$.

Similarly, contribution from the orbits of length $2 n \mathcal{L}$ is $\ell_{1} 2(-1)^{n} \cos k 2 \mathcal{L} n$. Taking into account contribution from the paths from $\mathbb{P}_{r}$ (starting by first going around the right loop), we obtain the following expression:

$$
\begin{aligned}
u(k) & =\frac{\mathcal{L}}{\pi}+\frac{1}{\pi}(\underbrace{\ell_{1}+\ell_{2}}_{=\mathcal{L}}) \sum_{n=1}^{\infty} 2(-1)^{n} \cos k 2 \mathcal{L} n \\
& =\frac{\mathcal{L}}{\pi}+\frac{\mathcal{L}}{\pi}\left(\sum_{m=1}^{\infty}\left(2 e^{i k 4 \mathcal{L} m}-e^{i k 2 \mathcal{L} m}\right)+\sum_{m=1}^{\infty}\left(2 e^{-i k 4 \mathcal{L} m}-e^{-i k 2 \mathcal{L} m}\right)\right) \\
& =\frac{2 \mathcal{L}}{\pi} \sum_{m \in \mathbb{Z}} e^{-i k 4 \mathcal{L} m}-\frac{\mathcal{L}}{\pi} \sum_{m \in \mathbb{Z}} e^{-i k 2 \mathcal{L} m},
\end{aligned}
$$

which coincides with the expression (25) obtained using the left hand side of trace formula (23).

The performed calculations show why the Aharonov-Bohm effect is not present if one of the fluxes is zero: contributions from the periodic orbits going with non-zero flux cancel each other. Conversely, if one of the fluxes is not zero, then the spectrum depends 
on the other flux, as can be seen from equation (16). Similar results hold even if one of the fluxes is an integer multiple of $\pi$. It is not so difficult to propose different examples of graphs, where similar effects are observed. Trace formulae, together with our explicit calculations, provide a methodology to construct such graphs.

\section{Acknowledgements}

The work of PK was partially supported by the Swedish Research Council (Grant D0497301) and ZiF-Zentrum für interdisziplinre Forschung, Bielefeld (Cooperation Group Discrete and continuous models in the theory of networks). The authors would like to thank Muhammad Usman for careful reading of the manuscript.

\section{References}

[1] Y. Aharonov, D. Bohm. Significance of electromagnetic potentials in quantum theory. Phys. Rev., 1959, 115, P. 485-491.

[2] G. Berkolaiko, P. Kuchment. Introduction to quantum graphs. Mathematical Surveys and Monographs, Amer. Math. Soc., 2013, 186 Providence, RI.

[3] B. Gutkin and U. Smilansky. Can one hear the shape of a graph? J. Phys. A, 2001, 34, P. 6061-6068.

[4] V.Kostrykin, R. Schrader. Quantum wires with magnetic fluxes, Dedicated to Rudolf Haag. Comm. Math. Phys., 2003, 237 (1-2), P. 161-179.

[5] T. Kottos and U.Smilansky. Periodic orbit theory and spectral statistics for quantum graphs. Ann. Physics, 1999, 274 (1), P. 76-124.

[6] P. Kurasov. Graph Laplacians and topology. Arkiv för Matematik, 2008, 46, P. 95-111.

[7] P. Kurasov. Schrödinger operators on graphs and geometry. I. Essentially bounded potentials. J. Funct. Anal., 2008, 254 (4), P. 934-953.

[8] P. Kurasov. Inverse problems for Aharonov-Bohm rings. Math. Proc. Cambridge Philos. Soc., 2010, 148 (2), P. 331-362.

[9] P. Kurasov. Quantum graphs: spectral theory and inverse problems. To appear in Birkhäuser.

[10] P. Kurasov and M. Nowaczyk. Inverse spectral problem for quantum graphs. J. Phys. A, 2005, 38 (22), P. 4901-4915.

[11] P. Kurasov and M. Nowaczyk. Geometric properties of quantum graphs and vertex scattering matrices. Opuscula Math., 2010, 30 (3), P. 295-309.

[12] O. Post. Spectral analysis on graph-like spaces. Lecture Notes in Mathematics, 2012, 2039, 431 p.

[13] J.-P. Roth. Le spectre du laplacien sur un graphe. Theorie du potentiel, Lecture Notes in Math., 1984, 1096, P. 521-539 [in French]. 